\title{
Bioengineered 3D models to recapitulate tissue fibrosis
}

Marta Sacchi $^{1,2}$, Ruchi Bansal ${ }^{+* 1}$, Jeroen Rouwkema ${ }^{+* 2}$

${ }^{1}$ Department of Biomaterials Science and Technology, Technical Medical Centre, Faculty of Science and Technology, University of Twente, Enschede, The Netherlands.

${ }^{2}$ Department of Biomechanical Engineering, Technical Medical Centre, Faculty of Engineering Technology, University of Twente, Enschede, The Netherlands.

tThese authors contributed equally.

*Correspondence: r.bansal@utwente.nl (R. Bansal), j.rouwkema@utwente.nl (J. Rouwkema)

M.Sacchi: ORCID: 0000-0003-4767-3889

R. Bansal: website: http://bansal-liverlab.com/

ORCID: 0000-0003-2470-5876

J.Rouwkema: website: www.vascularizationlab.com

Twitter: @JeroenRouwkema

ORCID: $\underline{0000-0001-9666-9064}$

Keywords: fibrosis, tissue models, screening, self-assembly, biofabrication

\begin{abstract}
Fibrosis, characterized by progressive tissue stiffening resulting in organ failure, is a growing health problem affecting millions of people worldwide. Currently, therapeutic options for tissue fibrosis are severely limited and organ transplantation is the only effective treatment for the end-stage fibrotic diseases with inherent limitations. Recent advancements in engineered 3D in vitro human disease mimic models, recapitulating the tissue pathophysiology, have provided unique state-of-the-art platforms for (i) the understanding the biological mechanisms involved in the disease pathogenesis, and (ii) high throughput and reproducible drug screening. This review will focus on the recent multidisciplinary developments made towards advanced 3D biomimetic fibrotic tissue (liver, kidney and lung) models that combine highly precision manufacturing techniques with high cellular functionality and biophysical (mechanical) properties.
\end{abstract}




\section{Fibrosis}

Fibrosis, characterized by an accumulation of excessive extracellular matrix (ECM) resulting in scar tissue and organ dysfunction, is a significant health problem affecting millions of people worldwide. With an increasing prevalence of obesity and diabetes, the incidence of fibrotic diseases and subsequently the number of fibrosis-related deaths will rise exponentially. Fibrosis is a common outcome of many chronic diseases in different tissues, including liver, kidneys and lungs. Currently, there are no therapies available to reverse chronic fibrosis. Organ transplantation is the only option available for these patients, but organ shortage and chronic rejection limit the feasibility. The persistence of this unmet clinical need is due to the complexity of the disease process that involves numerous cell types and signaling pathways. Lack of reliable and reproducible, in vitro models recapitulating the pathophysiology of tissue fibrosis, has partially hampered the development of anti-fibrotic drugs. Therefore, in past years, tremendous advancements have been made towards the development of bioengineered fibrosis models, mimicking human disease pathophysiology, as reviewed here. Even though fibrosis plays an important role in many tissues, including heart [1] and skin [2], this review will focus on fibrotic liver, lung and kidney tissue models.

Tissue fibrosis is a complex process, mainly characterized as a wound healing process triggered by chronic tissue injury (Box $\mathbf{1}$ and Figure I). Owing to this complexity, development of therapies has been challenging. Further, therapies proved potentially effective in animal fibrosis models, failed in clinical trials suggesting disparity between animal models and human. This led to the development of human disease mimicking in vitro models advancing from 2D monocultures/cocultures to self-assembled 3D spheroids and patient-derived organoids; from 3D-bioprinted and 3D-vascularized, bioreactor-based and micro-fluidically perfused organ-on-a-chip to tissue-based models including decellularized tissue scaffolds, precision-cut tissue slices (PCTS), and bioengineered artificial organs (Figure 1, Key Figure and Table 1). These models recapitulating multi-phenomenal tissue fibrosis, will not only provide a versatile tool for disease modeling, but will also aid in drug discovery by high-throughput drug screening in developed physiological in vitro disease models.

\section{2D models}

Two-dimensional (2D) models of fibrosis are still widely used as simple and reliable platforms for understanding the fundamental underlying disease mechanisms, and for high-throughput screening of pro- and anti-fibrotic compounds. 2D platforms provide several advantages such as ease-of-handling, multiplexing, high-reproducibility, single-cell imaging and profiling (genomic, transcriptomic and proteomic) under different culture conditions [3], based on which several fibrotic cellular markers have been identified.

Typical 2D cultures rely on the activation of primary rodent or human fibroblasts in monolayer on a tissue culture plastic (TCP). Primary fibroblasts, upon culturing on TCP or TGF- $\beta$ treatment, transdifferentiate into a highly proliferative, contractile and ECM-producing myofibroblasts (MFs) resembling their respective in vivo counterpart [4]. In addition, single- 
cell analyses are widely used to isolate and characterize lineage-mapped MFs. Such techniques, based on single-cell RNA sequencing and transcriptome analysis, reveal the underlying molecular reprogramming mechanisms and unravel the fibroblast landscape in fibrotic diseases [5] [6]. However, planar 2D culture platforms do not reproduce physiopathological tissue fibrosis. Comparison of gene expression profiles demonstrated that $2 \mathrm{D}$ culture activated hepatic stellate cells (HSCS) do not recapitulate the in vivo activated HSCS [3]. Furthermore, TCP is extremely stiff (ranges in GPa) compared to in vivo conditions (ranges in $\mathrm{kPa}$ ), suggesting that the mechanical environment in which these in vitro experiments are normally performed does not replicate tissue mechanics, which strongly influences cellular phenotype (Box 1). It has, for instance, been demonstrated that increasing ECM stiffness increases latent TGF- $\beta 1$ activation by MFs contraction [7]. Another major limitation of 2D models is their inability to mimic the 3D fibrotic microenvironment leading to physiologically irrelevant cellular behavior.

The physiological relevance of 2D platforms can be improved by: i) tuning the mechanical environment; ii) defining specific surface pattern on which cells can adhere and organize; iii) introducing 2D co-cultures. Studies using mechanically tunable substrates revealed that MFs differentiation increases with increasing ECM stiffness. Using a tunable PDMS substrate, Balestrini and coworkers showed increased $\alpha$-SMA expression, indicative of MFs activation, on $100 \mathrm{kPa}$ stiff substrates after 1 week of culture as compared to $5 \mathrm{kPa}$ soft substrates where they remain quiescent [8]. These ranges corresponded to the mechanical stiffness of a fibrotic lung tissue (20-100kPa) and a normal lung parenchyma (1-5kPa) [9].

A wide range of topographical surface patterns, defined as $2.5 \mathrm{D}$ cultures, have been introduced to reciprocate the physiological ECM and cell organization [10]. Furthermore, to increase complexity, a large number of $2 \mathrm{D}$ and $2.5 \mathrm{D}$ co-cultures have been designed. Several studies have demonstrated that the direct juxtacrine and indirect paracrine interactions influencing cellular phenotype [11]. Pulmonary epithelial air-liquid interface (ALI) culture, for instance, interfaced with lung microvascular endothelial cells and fibroblasts. These cocultures have enabled investigation of pathological paracrine signaling associated with different cell types $[12,13]$.

\section{3D models}

Even though 2D models have their merits, these models are not able to replicate the physiological 3D tissue environment. Similar to in vivo conditions, 3D models enable cell-cell and cell-matrix interactions, cellular migration, chemotaxis, traction and integrin adhesions, in all three planes, and replicate soluble growth factor gradients supporting cellular differentiation and maturation.

\subsection{Self-Assembled 3D systems}

Perhaps the simplest 3D models are those that are attained by cellular self-assembly. Upon the combination of multiple cells in suspension or in a physically constraint environment like a micro-well or hanging drop system, the cells aggregate to form 3D cellular structures. The 
lowest level of complexity is attained with cell aggregates composed of adult tissue cells or stem cell population, also referred to as spheroids [14]. Using a hanging-drop method, Bell and colleagues [15], have established and characterized phenotypically stable spheroids composed of primary human hepatocytes (PHH) and non-parenchymal cells (NPCs) [1NPC:2PHH]. Interestingly, these spheroids maintained proteomic levels for at least 5 weeks similar to those in the liver and retained inter-individual variability. In another study, Leite and colleagues [16] developed spheroids of HepaRG hepatocytes and HSCs [1HepaRG:2HSCs], using low attachment 96 well-plates, that displayed the fibrotic features such as HSCs activation upon single or repeated exposure (14d) to pro-fibrotic compounds (allyl alcohol and methotrexate). Coll and coworkers [17] developed highly reproducible iPSCs (induced pluripotent stem cells)-differentiated functional HSCs that remain quiescent when maintained as 3D spheroids with HepaRG and became activated in response to hepatocyte toxicity resembling in vivo human HSCs, thereby generated a powerful tool for studying HSCS development, disease modeling and drug toxicity screening. Based on these HSCs activation models, the biotech company InSphero has developed a standardized system of primary human liver spheroids including Kupffer cells amenable for anti-fibrotic drugs screening in a reproducible high-throughput system [18].

Organoids are more complex self-assembling structures of organ-specific cell types developed from stem cells or organ progenitor cells that follow sequential tissue developmental paradigms similar to in vivo $[19,20]$. As opposed to spheroids, organoids generally better replicate tissue shape and organization, and can contain multiple cell types that are distributed physiologically. Based on cell source, two different types of organoids can be distinguished; induced pluripotent stem cells (iPSCs)- and adult stem cells (ASCs)-derived organoids (Reviewed in [19, 21]).

iPSCs organoids form complex organ structures following typically developmental processes and can be used to study disease development. Besides disease modeling, organoids provide a unique platform for high-throughput drug screening, personalized medicines, toxicology studies and gene therapy. However, the development of iPSC-organoids is time-consuming, due to limited cell expansion and maturation abilities. On the other hand, ASCs-derived organoids recapitulate adult tissue repair instead of development. Hence, only tissues with regenerative capacity such as the epithelium, are feasible for this approach. ASCs-derived organoids form less complex structures compared to iPSCs, but they can undergo several expansion passages within a short period of time, and are genetically stable, which makes them highly suitable for personalized gene-editing approach based on CRISPR-genomic differentiation.

Sachs and coworkers [22] created long-term expanding human airway organoids containing basal, multi-ciliated and secretory cells derived from lung cancer resections and metastasis biopsies from cystic fibrosis patients, showing a clear resemblance between in vitro cultured organoids and human disease pathophysiology. Wilkinson and colleagues [23] generated selfassembled organoids with fetal lung fibroblasts, seeded on functionalized alginate hydrogel 
spheres using a rotational bioreactor. Upon TGF- $\beta 1$ incubation, these lung organoids depicted features of tissue scarring similar to IPF, and recapitulated lung microenvironment when cultured with endothelial and epithelial cells. This showed that these organoids don't only recapitulate tissue histology, but can also replicate a physiological tissue response. Comparably, Huang and coworkers [24] generated 3D lung organoids via directed differentiation of developmental iPSCs into functional respiratory airway epithelial cells. Using CRISPR/Cas9 gene editing, HPS-associated interstitial pneumonia 1 (HPS1) $/$ - 3D lung organoids were developed with HPS-associated interstitial pneumonia (HPSIP) fibrotic disease phenotype, clinically similar to IPF [25]. Furthermore, IL-11 upregulation was observed in $\mathrm{HPS1}^{-/}$fibrotic organoids and IL-11 deletion prevented fibrosis development, demonstrating the efficiency of such a culture system in modeling fibrotic lung disease and identifying therapeutic targets [25].

Takasato and colleagues [26] developed iPSCs-derived organoids containing cells from multiple kidney lineages (comprising mesangial cells and pericytes), resulting into a promising mini-kidney model resembling the first trimester human kidney. Comparably, long-term expanding primary kidney tubular epithelial organoids referred as 'tubuloids' resembling proximal and distal nephron segments were developed [27]. These were able to recapitulate kidney diseases, adopted a tubular conformation and displayed active transport when cultured on a microfluidic organ-on-a-chip device.

Recently, by combining the protocol developed by Takasato with the NovoGen Bioprinter MMX technology, the biotech company Organovo has developed a robust platform for pharmaceutical drug testing. The inclusion of the bioprinter has allowed for the automated and scaled fabrication of human iPSC-derived kidney organoids without compromising their functionality [28].

These 3D self-assembled systems therefore recapitulate numerous biological parameters including spatial cellular organization, cell-cell and cell-matrix interactions, and tissue-specific physiological functions. However, their future application is limited as these organoids resemble the primary substrate of disease, but lack mechanical cues, immune components, and perfused vasculature. Hence, even though these self-assembled systems can potentially model the cellular response to drugs, they are unlikely to replicate physiological disease progression and lack the capacity to model the infiltration of drugs via the blood.

\subsection{D biofabrication}

While organoids reproduce cellular organization using natural developmental processes, a precisely controlled process is often imperative, either to create a more physiological tissue organization, or to allow for a directed structural perturbation to investigate tissue response. In fibrosis modelling, the 3D tissue microenvironment is crucial to recapitulate typical fibrogenic features. The tissue-specific micro-environmental compartmentalization, such as patterns formed by collagen deposition or tissue stiffness, can provide more accurate insight into disease progression. By precisely positioning biological materials and living cells within 3D 
bio-fabricated tissue constructs can mimic tissue phenotype and functionality, with high throughput capability, customized geometries, and tunable mechanical properties.

Biofabrication technologies commonly used are extrusion [29], light-assisted (DLP and TPP) [30], inkjet (droplet-based) [31], and laser-induced forward transfer (LIFT) bioprinting [32]. Each technique has its pros and cons with respect to cell viability, printing resolution, structural integrity and materials (mostly biocompatible), resulting in constructs that to a varying extent resemble the mechanical and functional properties of fibrotic tissue. For detailed information regarding the different 3D bioprinting techniques, the reader is referred to previous reviews $[33,34]$.

Using extrusion-based printing, Nguyen and colleagues [35] developed 3D tissue constructs comprising parenchymal (primary human hepatocytes) and non-parenchymal (HSCs and ECS) cells with long-term functionality and exposure to different fibrogenic compounds induced liver injury [36]. Although tissue architecture did not recapitulate the native hepatocyte structure of the liver, the pattern of collagen deposition, cytokine production and fibrosisrelated gene expression profiles resembled multiple modes of liver injury. This model therefore replicated partial fibrogenesis mechanisms and could help to identify different druginduced liver injury pathways. Utilizing this 3D bio-printed model, Organovo has demonstrated its feasibility as high throughput model for pharmaceutical drug screening in the preclinical evaluation of certain anti-fibrotic drugs [37].

Ma and coworkers [38] developed a novel 3D-hydrogel-based triculture model which combined physiologically relevant cells with a liver-specific 3D microarchitecture. In particular, using DLP based bioprinting, hiPSC-derived hepatic progenitor cells (hiPSC-HPCs), endothelial and adipose-derived stem cells were embedded in a 3D hexagonal hydrogel construct with controlled stiffness, resembling the shape of a liver lobule. Cells reorganized and realigned into the hexagonal structure, maintained high cell viability and enabled hiPSC-HPCs maturation (Figure 2).

The biofabrication of the alveoli for development of lung and airway models on the contrary, remains a technical challenge due to their size scale and complexity [39]. In this aspect, photodegradable microspheres made of poly(ethylene glycol) (PEG) seeded with alveolar epithelial cells were developed, which resemble hollow epithelial cysts on the scale of alveoli [40]. Although these constructs insufficiently replicated a hierarchical structure, it has been speculated that these cell-laden microspheres can be combined with 3D-bioprinted alveoli architecture models [41] (Figure 2). In another study, membranous human lung microtissues were developed whereby the key biomechanical events occurring during lung fibrosis including progressive stiffening, alveolar tissue contraction accompanied with lung function decline was recapitulated. In addition, the anti-fibrotic drugs were evaluated for therapeutic efficacy suggesting the usability of this model for drug testing [42] (Figure 2).

In order to provide a highly tissue-specific microenvironment, specific-organ-derived decellularized ECM (dECM)-based bio-inks have been developed [43] such as a photo-cross 
linkable porcine kidney ECM-derived bio-ink for renal tissue bioprinting [44]. By combining renal dECM with different hydrogel materials, such as gelatin, hyaluronic acid and glycerol, a printable ink was developed combining primary human kidney cells, including proximal and distal tubular epithelial cells (PTECs/DTECs) and podocytes. The 3D-bioprinted construct was proven to provide structural integrity as well as a tissue-specific microenvironment.

Despite the successful studies, 3D-bioprinted organs have several challenges that must be overcome e.g. bioprinter technology (resolution and speed), biomaterials (biological versus synthetic), cell source (iPSCs versus other cells) and vasculature (for oxygen and nutrient supply).

\subsection{Microfluidic bioreactors and organ-on-a-chip technologies}

Although organoids or 3D-bioprinted constructs offer great potential, fluid-flow and active mechanical stimulation are not recapitulated in these systems. By combining cells, organoids, or 3D-bioprinted tissues with organ-on-chip technology, (interstitial) fluid flow, mechanical strain and hydrostatic pressures, which play a central role in fibrosis pathogenesis, can be integrated in the models.

Homan and coworkers created a 3D-perfusable human renal proximal tubule lined with PTECS embedded in ECM in a tissue chip, that showed a long-term maintenance of PTECs phenotype and function, forming a tissue-like epithelium and recapitulating in vivo microarchitecture [45]. To mimic the perisinusoidal space of the liver, a bioreactor consisting of two plates and a porous membrane was seeded with human umbilical vein endothelial cells (HUVECs) and activated HSCs (LX-2), and subjected to controlled shear stress by applying flow over the HUVECs. This demonstrated that HUVECs stimulated with shear stress inhibit HSCs via paracrine factors [46]. In another approach, Jeong and colleagues constructed a selforganizing micro-honeycomb structures using natural collagen-Matrigel matrix with hepatocytes and HUVECs spheroids for creating a complex-shape tunable 3D liver model mimicking liver ultrastructure and liver function [47].

Stucki and coworkers developed a 'breathing' lung-on-a-chip model with human alveolar epithelial cells and lung endothelial cells, with passive medium flow and cyclic strain, reproducing unique features of the alveolar microenvironment including breathing motion, the air-blood barrier, and the air-liquid interface [48]. Mondrinos and colleagues generated a human organ fibrosis chip by encapsulating fibroblasts within collagen hydrogels inside the lung-on-chip device. Chemically induced fibrotic responses were amplified by perfusing monocyte-derived macrophages and cytokines promoting M2 macrophage phenotype [49]. Recently, a 3D-bioengineered pulmonary fibrotic (Eng-PF) tissue was developed utilizing silk collagen-I hydrogels seeded with airway epithelial and microvascular endothelial cells, in combination with a Flexcell ${ }^{\circledR}$ bioreactor system [50]. Eng-PF tissue modeled fibroblastic features such as epithelial injury, myofibroblast differentiation, and enabled screening of antifibrotics. 
Zhou and coworkers developed a microfluidic and compartmentalized chip device that reproduces the fluidic and $3 \mathrm{D}$ microenvironment of the renal proximal tubule [51]. Using immortalized human proximal tubule cells, the epithelial-to-mesenchymal transition involved in the development of renal fibrosis could be recapitulated. Using similar microfluidic device, Kim and colleagues were able to expose kidney epithelial cells to controlled doses of gentamicin and to provide cells with physiological shear stress [52]. It was demonstrated that sub-lethal doses of gentamicin led to cell injury, causing reduction in tight junctional proteins and increase in the expression of kidney injury marker 1 . While this study only focused on gentamicin effects, the described method has the potential to be used for a wide range of drug-induced nephrotoxicity studies.

Despite the promising application for drug toxicity studies, many factors still remain to be addressed. For example, the limited cell sources and the maintenance of their long-term functionality within the system. Furthermore, a single organ-on-chip model is unable to resemble the main processes involved in pharmacokinetics such as adsorption and drug distribution.

\subsection{Vascularized models}

An organized and endothelialized vascular network within a bioengineered model is important for providing a physiological microenvironment, but also a physiological selective vascular barrier controlling the molecular transport from the vessels to the surrounding tissue [53]. Furthermore, tissue constructs generated in vitro require a constant flow of oxygen and nutrients, and a route to remove wastes, to maintain tissue viability and functionality. However, although the need for a vascular network within a fibrotic tissue model is clear, the engineering and integration of such networks in in-vitro models remains a challenge.

$3 \mathrm{D}$ bioprinting represents a valuable tool to address challenges in vascular tissue engineering with the versatility and flexibility to fabricate various designs from a wide range of biomaterials and the precision to control microscale features [54]. Kolesky and colleagues adopted a nozzle-based 3D printer for developing 3D cell-laden vascularized tissues that exceed $1 \mathrm{~cm}$ in thickness and can be perfused on chip for long periods of up to 6 weeks [55]. Parenchyma, stroma and endothelium were integrated within a single thick tissue by coprinting multiple inks composed of human mesenchymal stem cells and neonatal dermal fibroblasts with a customized ECM alongside embedded vasculature lined with HUVECs. In another approach, Zhu and coworkers used a rapid DLP-based bioprinting to create prevascularized tissue construct with notably high speed, resolution and scalability [54] for integration within (micro)fluidic devices or bioreactors to simulate blood flow and diffusion.

Recently, Homan and colleagues reported an in vitro method for culturing iPSC-derived human kidney organoids under flow on milli-fluidic chips [56]. They demonstrated that kidney organoids under fluidic shear stress exhibited highly vascularized tubular and glomerular compartment with maturation of TECs and podocytes. Vascularized kidney organoids 
emulated key kidney functions, even though the method did not ensure the perfusability of the microvascular networks (Figure 2). In another study, 3D-vascularized proximal tubules (VasPTs) were fabricated, using epithelium and endothelium embedded in engineered ECM, that exhibited active reabsorption of solutes via tubular-vascular exchange and vectoral transport of solutes, thereby providing a unique platform for kidney disease modeling and drug testing [57] (Figure 2). Similarly, 3D-vascularized liver organoids composed of iHEP cells, endothelial cells and hepatic dECM hydrogel cultured in a microfluidic system with a continuous dynamic flow exhibited liver-specific functions [58] (Figure 2).

Different cell types are involved in the formation of a healthy vasculature. Endothelial cells (ECS) form a selective and functional monolayer barrier between the blood and the tissue compartment. Pericytes and vascular smooth muscle cells (VSMCs) play an important role in stabilizing ECs tubes and regulating vascular tone. Where the studies mentioned previously only used ECs for the vascular networks, reports also exists where all the cellular components were generated from hiPSCs and successfully integrated in microfluidic chips and bioreactors, forming lumenized vessel structures and capillary networks [59] [60]. However, the question remains whether hiPSCs-derived vascular cells can actually acquire mature characteristics similar to primary cells in engineered microfluidic devices, thus fully replicating the natural barrier function [61].

\subsection{Physiological tissue-based models}

By combining bioprinting using organ-specific bio-inks with organ-specific functional cells and perfused vascular networks, a biomimetic organotypic model can be attained that closely resembles the in vivo situation. However, this approach is highly complex. Therefore, an alternative approach to achieve a physiological tissue model has been developed, referred to as precision-cut tissue slices (PCTS). PCTS are obtained by cutting fresh tissue into 200-250 $\mu \mathrm{m}$ thick slices [62]. The major drawback of this system is that the slice preparation results in 2 -cut surfaces that trigger a repair and regenerative response e.g. HSCs activation in liver slices within 96h [63].

In an interesting study, it was demonstrated that a mixture of pro-fibrotic factors, proinflammatory cytokines and signaling molecules (TGF- $\beta$, TNF- $\boldsymbol{\alpha}$, platelet-derived growth factor) could induce early fibrosis-like characteristics (except $\alpha$-SMA induction) in human precision-cut lung slices (PCLS) derived from patient without IPF [64]. Similarly, precision-cut kidney slices (PCKS) derived from human cortical tissue from tumor nephrectomies [65] demonstrated TGF- $\beta 1$-induced renal fibrogenesis characterized by an expression of multiple fibrosis markers, including $\alpha$-SMA. PCKS showed maintenance of renal tissue architecture and phenotype (up to 48h), demonstrating its application as promising renal fibrosis model.

Paish and coworkers described a novel bioreactor system with extended metabolic activity and functional longevity of precision-cut liver slices, to at least 6-days, without hepatocellular stress and significant spontaneous fibrogenesis [66]. This provided an efficient model for modeling fibrogenesis and testing potential anti-fibrotic drugs. 
In an alternative approach, PCTS can be decellularized, leaving the organized ECM and, depending on the decellularization method, proteins such as growth factors. Interestingly, acellular patient-derived scaffolds can retain unique, individual protein composition and structure of their respective disease pathologies (up to 1 month) [67]. Primary human fibroblasts seeded on acellular lung scaffolds derived from IPF patients, displayed myofibroblasts phenotype with increased $\alpha$-SMA expression. Apart from specific cell-ECM interactions, acellular scaffolds potentially model the extracellular microenvironment of the diseased tissue, including matrix sequestered growth factors involved in disease onset and pathogenesis [68]. However, scaffolds derived from tissues represent the end-stage disease and are often reseeded with only one or two cell types, thus incompletely modelling cell-cell interactions.

\section{Applications and future outlook}

Recent advancements have enabled recapitulation of different aspects of human fibrotic diseases, which will significantly contribute to early-stage drug discovery. However, due to the disease complexity, modeling of tissue fibrosis remains challenging, and a clear cut recipe for the ideal model does not yet exist (see Outstanding Questions). Current bioengineered systems often lack the topographical multicellular organization, perfusable (micro)vasculature, multicellular response to injury, and organ-specific immune response, which will need to be integrated into the optimal new generation fibrotic tissue models. However, before designing such complex models, one should define what level of complexity is actually needed in a specific model for a specific application. Even though the addition of complexity, e.g. by including a vascular network or immune cells, will make the tissue model more physiological, it is also likely to result in models that are harder to operate, control and evaluate. As such, tissue models should be as complex as needed to answer specific questions, but also as simple as possible to allow for an efficient usage.

Furthermore, the progressive development of robust hiPSC's differentiation approaches are highly promising and will facilitate development of patient-specific fibrotic models for personalized disease modeling and personalized medicines. However, for successful future implementation, strategies for the acquisition of patient-specific iPSC's need to be further optimized. Imperatively, integration of bioreactors and micro/milli-fluidic devices can improve the longevity of bioengineered models and provide high level of control over cellular microenvironment [69]. This control will facilitate the use of guided self-organization for the formation of complex spatially organized tissue models, which is a strong alternative to technologies like 3D-bioprinting where tissue remodeling after fabrication is often difficult to predict and control. Additionally, a temporal control over tissue development could allow for the replication of different stages of fibrosis, resulting in a deeper understanding about disease progression and the possibility to test different fibrosis stage-based treatment options.

Despite the shortcomings, recent advancements in engineered fibrotic tissue- and organ models do represent promising platforms for efficiently recapitulating the biological 
mechanisms involved in fibrosis pathogenesis, providing versatile tools for disease modelling and drug testing in clinically relevant disease models.

\section{Acknowledgements}

JR received financial support from the European Research Council (ERC) under the European Union's Horizon 2020 research and innovation programme (724469). 


\section{BOX 1}

\section{Mechanisms of pathological fibrosis}

Fibrosis is a wound healing process initiated by chronic tissue injury followed by chronic inflammation and ECM deposition, ultimately leading to organ failure (Figure I). Chronic injury to epithelial cells triggers the release of pro-inflammatory factors instigating the recruitment and activation of immune cells in the damaged tissue. Subsequently, these immune cells release pro-fibrotic factors, such as cytokines, chemokines and growth factors that cause the trans-differentiation of quiescent fibroblasts into activated myofibroblasts (MFs), identified by alpha-smooth muscle actin ( $\alpha$-SMA) expression. These cells secrete a vast amount of ECM components such as collagens resulting in tissue scarring and fibrosis [70].

MFs originate from different sources including tissue-resident mesenchymal cells, epithelial and endothelial cells via epithelial-/endothelial-mesenchymal (EMT/EndMT) transition, pericytes and bone marrow (BM)-derived mesenchymal stromal cells (MSCs) [71, 72]. In liver, the primary source of MFs are hepatic stellate cells (HSCs) and portal fibroblasts [73]. Upon chronic injury, scar tissue deposition replaces the hepatocytes, causing the loss-of-liver function [74]. In kidneys, the main sources are pericytes, interstitial fibroblasts, and glomerular mesangial cells. Upon chronic injury, glomerular and tubulo-interstitial ECM accumulation lead to loss-of-organ architecture and function leading to chronic kidney disease. In lungs, the major sources are interstitial fibroblasts, BM-derived fibrocytes, mesothelial cells and epithelial alveolar cells. Chronic pulmonary injury results in Idiopathic Pulmonary Fibrosis (IPF) [75]. Furthermore, these tissues have significant amount of blood flow through specialized vessels including the sinusoids in the liver, glomerulus in the kidney, and pulmonary vessels in the lung. Altered flow through these vessels could be the cause and result of tissue remodeling and fibrosis leading to pathological angiogenesis [76].

Although most research has focused on MFs to understand disease mechanisms and design drug targeting approaches, other components such as immune (especially macrophages) and endothelial cells, soluble molecules (chemokines, cytokines and growth factors, notably TGFß) [77], hypoxia, matrix-stiffness, mechanical strain/shear stress, vascular and interstitial flow, have also been recognized to play an important role [78]. MFs cause ECM reorganization and contraction affecting cellular behavior in response to biochemical and mechanical stimuli [7] [79]. Importantly, mechanical stimulus driven by physical microenvironment or cellular mechanosensing by different mechanisms involving calcium, mechanosensitive-sensitive ion channels, matrix mechanosensors e.g. integrins, transcription mechanosensitive factors e.g. YAP/TAZ, myocardin-related transcription factor (MRTF), is an important mechanism in organ fibrosis [80]. Fibrosis evolves from a concerted interaction between different cell types with tissue microenvironmental signals, involving autocrine, paracrine, juxtacrine, endocrine, angiocrine, mechanocrine and neuroendocrine signaling. 
1 Table 1: advantages and disadvantages of current classes of fibrotic tissue models

\begin{tabular}{|c|c|c|c|c|}
\hline Model & Uses & Advantages & Disadvantages & References \\
\hline 2D culture & $\begin{array}{l}\text { a. Screening of anti-fibrotic agent } \\
\text { and lineage-mapping studies } \\
\text { b. Cell migration and } \\
\text { mechanobiology studies } \\
\text { c. Co-culture studies to investigate } \\
\text { paracrine interactions }\end{array}$ & $\begin{array}{ll}\text { - } & \text { High throughput } \\
\text { - } & \text { Ease of handling, multiplexing } \\
\text { - } & \text { Single-cell imaging and analysis } \\
\text { - } & \text { Gene expression profiling } \\
\text { - } & \text { Cell-cell and cell-matrix interaction } \\
\text { - } & \text { Tunable stiffness fabrication }\end{array}$ & $\begin{array}{ll}\text { - } & \text { Lack of complexity } \\
\text { - } & \text { Cell-ECM and cell-cell } \\
\text { interaction only in X-Y plane } \\
\text { - } \quad \text { Physiological irrelevant cell } \\
\text { behavior and response due to } \\
\text { high matrix stiffness }\end{array}$ & $\begin{array}{l}{[7,10]} \\
\text { a. Liver }[3,4] \text {, lung } \\
{[5], \text { kidney [6] }} \\
\text { b. Lung }[8,9] \\
\text { c. Liver }[11] \text {, lung } \\
{[12,13]}\end{array}$ \\
\hline 3D spheroids & $\begin{array}{l}\text { a. Drug screening and toxicity } \\
\text { studies } \\
\text { b. Mechanistic studies } \\
\text { understanding the in vivo and } \\
\text { patient mimic fibrotic } \\
\text { microenvironment }\end{array}$ & $\begin{array}{ll}\text { - } & \text { Self-assembled 3D cellular organization } \\
\text { - } & \text { 3D cell-cell and cell-matrix interaction } \\
\text { - } & \text { Relatively easy to fabricate } \\
\text { - } & \text { High throughput }\end{array}$ & $\begin{array}{ll}\text { - } & \text { Simplified architecture } \\
\text { - } & \text { Size variation and } \\
\text { heterogeneity } \\
\text { - } \quad \text { Lack of vasculature }\end{array}$ & $\begin{array}{l}{[14]} \\
\text { a. Liver }[15,16] \\
\text { b. Liver }[17]\end{array}$ \\
\hline 3D organoids & $\begin{array}{l}\text { a. Adult stem cells (ASCs) derived } \\
\text { organoids: disease modeling. } \\
\text { b. Pluripotent stem cells (iPSCs) } \\
\text { derived organoids: fibrosis } \\
\text { development modeling } \\
\text { c. Drug screening and toxicity } \\
\text { studies }\end{array}$ & $\begin{array}{ll}\text { - } & \text { Self-assembled 3D cellular organization } \\
\text { - } & \text { Patient-derived (ASCs and hiPSCs): mimic } \\
\text { complexity and architecture } \\
\text { - } \quad \text { Genetical stability } \\
\text { - } & \text { Personalized disease modeling and } \\
& \text { treatment, and CRISPR genome editing }\end{array}$ & $\begin{array}{l}\text { - } \quad \text { Lack of vasculature/perfusion } \\
\text { - } \quad \text { Limited expansion } \\
\text { - } \quad \text { Time-consuming: long-term } \\
\text { culture } \\
\text { - } \quad \text { Less amenable to high } \\
\quad \text { throughput screening }\end{array}$ & $\begin{array}{l}\text { [19-21] } \\
\text { a. Lung }[22,23] \text {, } \\
\text { kidney }[26] \\
\text { b. Lung }[24] \text {, } \\
\text { kidney }[27] \\
\text { c. Lung }[25]\end{array}$ \\
\hline 3D bioprinting & $\begin{array}{l}\text { a. Fabrication of 3D biomimetic } \\
\text { scaffold similar to in vivo situation } \\
\text { b. Replication and study of induced } \\
\text { fibrogenesis mechanisms at the } \\
\text { cellular and physiological level } \\
\text { c. 3D cell-cell and cell-ECM } \\
\text { interaction studies }\end{array}$ & $\begin{array}{l}\text { - } \quad \text { High throughput and long-term cultures } \\
\text { - } \quad \text { Precision control over size and shape } \\
\text { - } \quad \text { Tunability of mechanical properties } \\
\text { - } \quad \text { Cell phenotype, gene expression and } \\
\text { functionality preservation } \\
\text { - } \quad \text { Possibility to combine different types of } \\
\text { biomaterials, cells and growth factors }\end{array}$ & $\begin{array}{l}\text { - } \quad \text { Lack of vasculature/perfusion } \\
\text { - } \quad \text { Challenges with cell viability } \\
\text { - } \quad \text { Challenges with materials for } \\
\text { printing: biocompatibility and } \\
\text { mechanical properties } \\
\text { (structural integrity) } \\
\text { - } \quad \text { Difficult for high throughput }\end{array}$ & $\begin{array}{l}\text { [29-34, 43] } \\
\text { a. Liver [35], lung } \\
{[39,40]} \\
\text { b. Liver [36, 38], } \\
\text { lung [41, 42] } \\
\text { c. Kidney [44] }\end{array}$ \\
\hline $\begin{array}{l}\text { Bioreactors } \\
\text { and organ-on- } \\
\text { chip }\end{array}$ & $\begin{array}{l}\text { a. Studies of induced fibrogenesis } \\
\text { mechanisms at the cellular and } \\
\text { physiological level under dynamic } \\
\text { environment } \\
\text { b. Evaluating effects of drugs, due to } \\
\text { dynamic tissue microenvironment }\end{array}$ & $\begin{array}{l}\text { - } \quad \text { Replicate dynamic in vivo } \\
\text { microenvironment including } \\
\text { hydrodynamic and mechanical cues } \\
\text { - } \quad \text { Oxygen and nutrients supply and } \\
\text { removal of metabolic waste } \\
\text { - } \quad \text { Multiplexed with other 3D systems }\end{array}$ & $\begin{array}{l}\text { - } \quad \text { Lack of vasculature/perfusion } \\
\text { - Difficult to adapt for high } \\
\text { throughput }\end{array}$ & $\begin{array}{l}\text { a. Kidney }[45,52] \\
\text { liver }[46,47] \text {, } \\
\text { lung }[48-50] \\
\text { b. Kidney }[51]\end{array}$ \\
\hline
\end{tabular}


a. In vitro study of vascular remodeling and cell migration

b. Drug screening

\section{Decellularized}

tissue

matrices

Precision cut

tissue slices a. In vitro culture substrate mimicking native or fibrotic disease microenvironment

a. In vitro culture substrate mimicking native or fibrotic disease microenvironment
- $\quad$ Chemical transport and cellular diffusion

Enhanced cell/tissue maturation and organization

- $\quad$ Selective barrier for drug testing

- Multiplexing with microfluidic systems

Possibility to develop patient-specific vascularized model with iPSCs

- $\quad$ High throughput capacity

Replicate in vivo cell-matrix interaction

Restored native matrix stiffness

Restored native protein, growth factors and structure (depending on the process)

Long-term culture and storage $\left(-80^{\circ} \mathrm{C}\right)$

Dimensional tunability

- Accurate cell-matrix, cell-cell

interactions

Dimensional tunability

- High throughput capacity
Dimensional limitations

- $\quad$ Risk of clogging and bursting

during perfusion

Limited perfusability

- iPSCs maturation in

microfluidic devices

Accessibility to the tissue samples

Non-anatomical

microenvironment

Does not model cell-cell

interaction

ECM loss during procedure Represent end-stage disease

\section{Relatively short-term cultures}

- Repair and regenerative responses

Lack of intact immune system
[53-55, 59-61]

a. Kidney [56], liver [58]

b. Kidney [57]

a. Lung $[67,68]$

\section{$[62,63]$}

a. Lung [64], kidney [65], liver [66] 
4
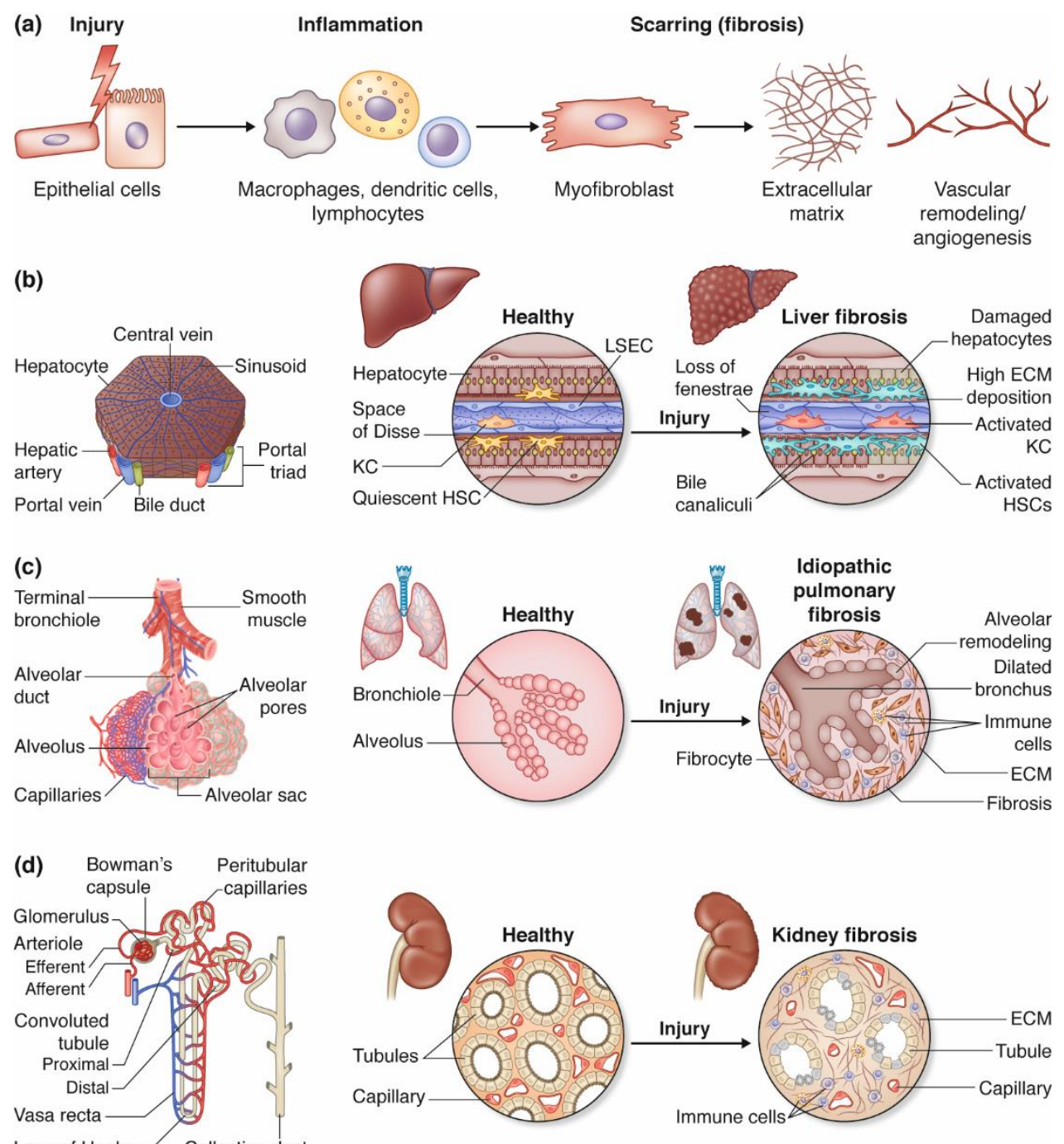

6 Figure I: Schematic illustration depicting tissue microstructures and fibrosis pathogenesis. (a)

7 Schematic diagram presenting general pathogenesis of tissue fibrosis. Injury to epithelial cells

8 instigate inflammation i.e. infiltration of immune cells. The inflammatory cells secrete cytokines and

9 growth factors which activate local fibroblasts and infiltrated mesenchymal cells into

10 myofibroblasts. Eventually, myofibroblasts secrete extracellular matrix (ECM) remodeling leading to

11 scarring of the tissue and vascular remodeling/angiogenesis. (b) Schematic representation of liver

12 lobule with changes in liver architecture during fibrosis. (c) Schematic diagram of lung alveoli and

13 changes in the lung architecture during fibrosis. (d) Schematic representation of kidney tubule with 14 changes in the kidney architecture during fibrosis. 


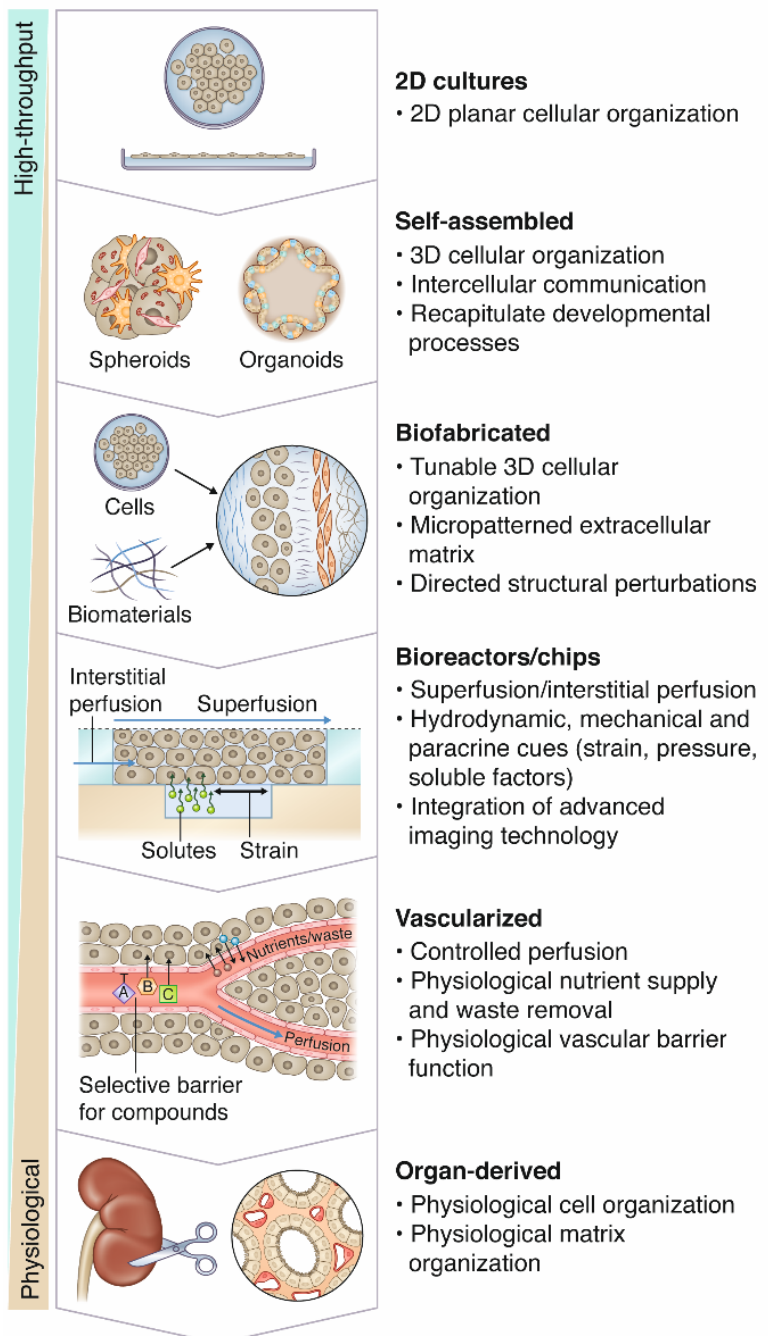

Figure 1: Schematic illustration depicting evolution of physiologically relevant models. Compared to the traditional two-dimensional (2D) cell cultures as monolayers on flat surfaces, threedimensional (3D) structures i.e. self-assembled spheroids and organoids form more complex 3D structures reflecting physiological cellular organization and intercellular communication and, can recapitulate physiological developmental processes. Several new technological advancements have been made over past years including biofabrication with tunable 3D cellular organization and micropatterned ECM allowing for directed structural perturbations; bioreactors/organ-on-a-chips with possibility of superfusion/interstitial perfusion integrated with hydrodynamic, mechanical and soluble paracrine cues, and advanced imaging technology; vascularized organs with opportunities for controlled perfusion, physiological vascular barrier function and physiological nutrient supply and waste removal to highly physiological cell and matrix organization in organ-derived microtissues including vascularized organoids and precision-cut tissue slices. Comparatively, models with increasing complexity and physiological relevance are not always applicable for high-throughput drug screening but provide in-depth knowledge about disease modeling and are useful for development of personalized treatments and provide insights about the translational potential of the novel drug molecules. 


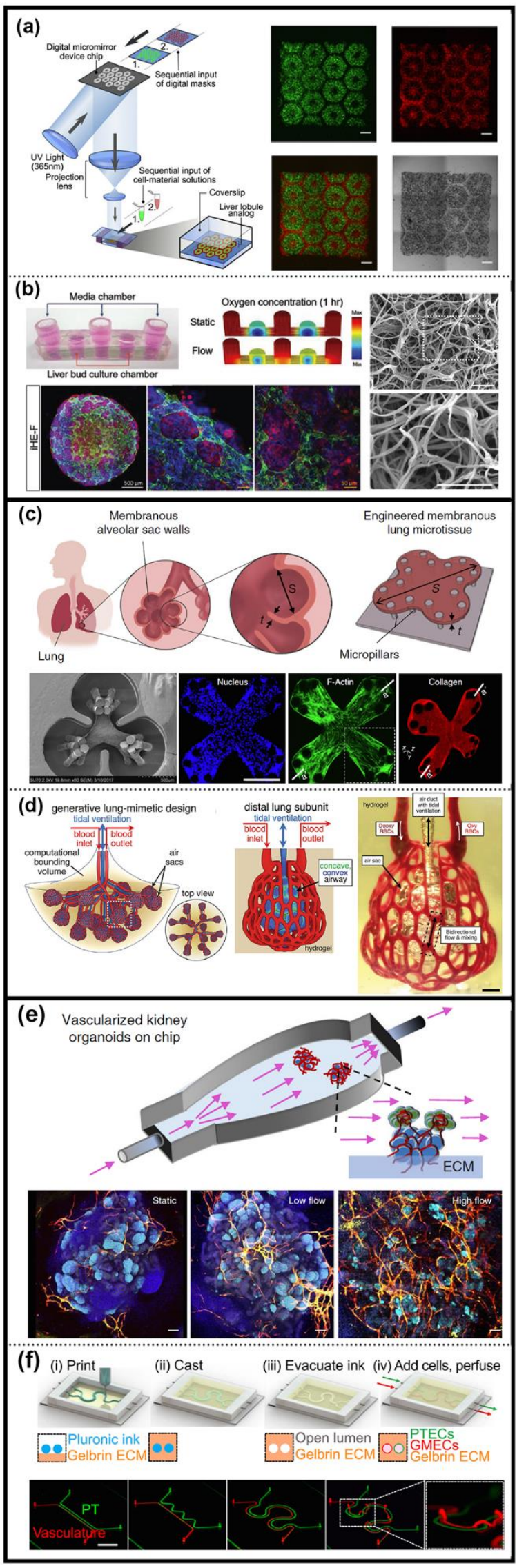

35 Figure 2: Illustration of 3D tissue models for disease modeling and drug testing: (a) A two-step 36 DMD-based 3D bioprinting of liver lobule structures (ref. 38). (b) 3D vascularized liver organoid 
37 composed of iHep cells and a decellularized liver extracellular matrix (LEM) cultured in a microfluidic 38 system (ref. 58). (c) Engineered lung microtissues that model the membranous morphology of the 39 alveolar sac wall (ref. 42). (d) 3D bioprinted vascularized alveolar lung-mimetic models with 40 multivascular networks and functional intravascular topologies (ref. 41). (e) Bioengineered 41 vascularized kidney organoids in a microfluidic system (ref. 56). (f) Design and fabrication of 3D 42 Vascular proximal tubular models (ref. 57). All images have been reproduced with permission from $43[38,58,42,41,56,57]$ respectively. 
Fibrosis: tissue stiffening as a result of excessive extracellular matrix deposition.

Extracellular matrix: network of extracellular macromolecules providing the structural and biochemical support for cells in a tissue.

Myofibroblasts: a cell that is in between a fibroblast and a smooth muscle cell in phenotype. Myofibroblasts deposit vast amounts of extracellular matrix and are involved in tissue contraction.

Parenchyma: the functional tissue of an organ as distinguished from the connective and supporting tissue.

Hepatocytes: the main functional cell type in the parenchymal tissue of the liver.

Hepatic stellate cells: when activated, the main cell type involved in liver fibrosis. Activated stellate cells are characterized by proliferation, contractility, and extracellular matrix deposition.

Organoids: self-organized three-dimensional tissue cultures that are derived from stem cells. Organoids contain multiple organ-specific cell types, which are spatially organized to replicate the organ organization, and are capable of replicating specific organ functions.

Transforming growth factor beta (TGF- $\beta 1$ ): growth factor which is mainly involved in the regulation of inflammatory processes.

Tumor necrosis factor alpha (TNF- $\alpha$ ): growth factor which promotes the systemic inflammatory response.

3D biofabrication: the use of additive manufacturing technology for the precise placement of biological materials and cells in 3D.

Organ-on-a-chip technology: 3-D cell culture platforms, based on microfluidic technology, that simulate the activities, mechanics and physiological responses of organs and organ systems.

Juxtacrine signaling: cell signaling dependent on direct cell-cell contact.

67 Paracrine signaling: cell signaling dependent on secreted factors.

CRISPR/Cas9 gene editing: technique with which genomic DNA can be edited efficiently and highly selectively.

Interleukin 11 (IL-11): a downstream cytokine of TGF- $\beta 1$ signaling, associated with fibrotic diseases.

Pericytes: cells that lies around and stabilize endothelial cells that line capillaries and venules.

Perisinusoidal space or space of disse: is the narrow space between a hepatocyte and a liver sinusoid

Kidney glomerulus: a network of small blood vessels (capillaries) that form the glomerular capillary bed and is located in the nephron in the kidneys.

Liver sinusoids: are a type of fenestrated/porous blood vessel that form the capillary bed in the liver parenchyma. 


\section{References:}

1. Hinderer, S. and Schenke-Layland, K. (2019) Cardiac fibrosis - A short review of causes and therapeutic strategies. Adv Drug Deliv Rev 146, 77-82.

2. Do, N.N. and Eming, S.A. (2016) Skin fibrosis: Models and mechanisms. Curr Res Transl Med 64 (4), 185-193.

3. De Minicis, S. et al. (2007) Gene Expression Profiles During Hepatic Stellate Cell Activation in Culture and In Vivo. Gastroenterology 132 (5), 1937-1946.

4. Seki, E. et al. (2007) TLR4 enhances TGF- $\beta$ signaling and hepatic fibrosis. Nature Medicine 13, 1324.

5. Xie, T. et al. (2018) Single-Cell Deconvolution of Fibroblast Heterogeneity in Mouse Pulmonary Fibrosis. Cell reports 22 (13), 3625-3640.

6. Duffield, J.S. (2014) Cellular and molecular mechanisms in kidney fibrosis. The Journal of clinical investigation 124 (6), 2299-2306.

7. Wipff, P.-J. et al. (2007) Myofibroblast contraction activates latent TGF- $\beta 1$ from the extracellular matrix. The Journal of Cell Biology 179 (6), 1311.

8. Balestrini, J.L. et al. (2012) The mechanical memory of lung myofibroblasts. Integrative Biology 4 (4), 410-421.

9. Hinz, B. (2012) Mechanical Aspects of Lung Fibrosis. Proceedings of the American Thoracic Society 9 (3), 137-147.

10. Mirbagheri, M. et al. (2019) Advanced cell culture platforms: a growing quest for emulating natural tissues. Materials Horizons 6 (1), 45-71.

11. Sawitza, I. et al. (2009) The niche of stellate cells within rat liver. Hepatology 50 (5), 1617-1624.

12. Epa, A.P. et al. (2015) Normal Human Lung Epithelial Cells Inhibit Transforming Growth Factor$\beta$ Induced Myofibroblast Differentiation via Prostaglandin E2. PloS one 10 (8), e0135266-e0135266.

13. Prasad, S. et al. (2014) Deficient repair response of IPF fibroblasts in a co-culture model of epithelial injury and repair. Fibrogenesis \& tissue repair 7, 7-7.

14. Fennema, E. et al. (2013) Spheroid culture as a tool for creating 3D complex tissues. Trends in Biotechnology 31 (2), 108-115.

15. Bell, C.C. et al. (2016) Characterization of primary human hepatocyte spheroids as a model system for drug-induced liver injury, liver function and disease. Scientific Reports 6, 25187.

16. Leite, S.B. et al. (2016) Novel human hepatic organoid model enables testing of drug-induced liver fibrosis in vitro. Biomaterials 78, 1-10.

17. Coll, M. et al. (2018) Generation of Hepatic Stellate Cells from Human Pluripotent Stem Cells Enables In Vitro Modeling of Liver Fibrosis. Cell Stem Cell 23 (1), 101-113.e7.

18. https://insphero.com/applications/diabetes-and-nash-discovery/liver/fibrosis/

19. Clevers, H. (2016) Modeling Development and Disease with Organoids. Cell 165 (7), 1586-1597.

20. Yin, X. et al. (2016) Engineering Stem Cell Organoids. Cell stem cell 18 (1), 25-38.

21. Drost, J. and Clevers, H. (2018) Organoids in cancer research. Nature Reviews Cancer 18 (7), $407-$ 418. 
22. Sachs, N. et al. (2019) Long-term expanding human airway organoids for disease modeling. The EMBO Journal 38 (4), e100300.

23. Wilkinson, D.C. et al. (2017) Development of a Three-Dimensional Bioengineering Technology to Generate Lung Tissue for Personalized Disease Modeling. Stem cells translational medicine 6 (2), 622-633.

24. Huang, S.X.L. et al. (2013) Efficient generation of lung and airway epithelial cells from human pluripotent stem cells. Nature Biotechnology 32, 84.

25. Snoeck, H.-W. et al. (2019) Modeling of fibrotic lung disease using 3D organoids derived from human pluripotent stem cells. bioRxiv, 538991.

26. Takasato, M. et al. (2015) Kidney organoids from human iPS cells contain multiple lineages and model human nephrogenesis. Nature 526, 564.

27. Schutgens, F. et al. (2019) Tubuloids derived from human adult kidney and urine for personalized disease modeling. Nature Biotechnology 37 (3), 303-313.

28.

https://organovo.com/wp-content/uploads/2019/07/2019-ISSCR-Organovo-posterWHiggins.pdf

29. Ozbolat, I.T. and Hospodiuk, M. (2016) Current advances and future perspectives in extrusionbased bioprinting. Biomaterials 76, 321-343.

30. Wang, Z. et al. (2018) A Novel, Well-Resolved Direct Laser Bioprinting System for Rapid Cell Encapsulation and Microwell Fabrication. Advanced Healthcare Materials 7 (9), 1701249.

31. Xu, T. et al. (2005) Inkjet printing of viable mammalian cells. Biomaterials 26 (1), 93-99.

32. Mézel, C. et al. (2010) Bioprinting by laser-induced forward transfer for tissue engineering applications: jet formation modeling. Biofabrication 2 (1), 014103.

33. Derakhshanfar, S. et al. (2018) 3D bioprinting for biomedical devices and tissue engineering: $A$ review of recent trends and advances. Bioactive Materials 3 (2), 144-156.

34. Ma, X. et al. (2018) 3D bioprinting of functional tissue models for personalized drug screening and in vitro disease modeling. Advanced Drug Delivery Reviews 132, 235-251.

35. Norona, L.M. et al. (2016) Editor's Highlight: Modeling Compound-Induced Fibrogenesis In Vitro Using Three-Dimensional Bioprinted Human Liver Tissues. Toxicological Sciences 154 (2), 354-367.

36. Nguyen, D.G. et al. (2016) Bioprinted 3D Primary Liver Tissues Allow Assessment of Organ-Level Response to Clinical Drug Induced Toxicity In Vitro. PloS one 11 (7), e0158674-e0158674.

37. https://organovo.com/wp-content/uploads/2019/01/Organovo-Poster-SOT-Mar2018.pdf

38. Ma, X. et al. (2016) Deterministically patterned biomimetic human iPSC-derived hepatic model via rapid 3D bioprinting. Proceedings of the National Academy of Sciences 113 (8), 2206.

39. Galliger, Z. et al. (2019) 3D bioprinting for lungs and hollow organs. Translational Research.

40. Lewis, K.J.R. et al. (2015) In vitro model alveoli from photodegradable microsphere templates. Biomaterials science 3 (6), 821-832.

41. Grigoryan, B. et al. (2019) Multivascular networks and functional intravascular topologies within biocompatible hydrogels. Science 364 (6439), 458.

42. Asmani, M. et al. (2018) Fibrotic microtissue array to predict anti-fibrosis drug efficacy. Nature Communications 9 (1), 2066. 
43. Garreta, E. et al. (2017) Tissue engineering by decellularization and 3D bioprinting. Materials Today 20 (4), 166-178.

44. Ali, M. et al. (2019) A Photo-Crosslinkable Kidney ECM-Derived Bioink Accelerates Renal Tissue Formation. Advanced Healthcare Materials 8 (7), 1800992.

45. Homan, K.A. et al. (2016) Bioprinting of 3D Convoluted Renal Proximal Tubules on Perfusable Chips. Scientific Reports 6, 34845.

46. Illa, X. et al. (2014) A novel modular bioreactor to in vitro study the hepatic sinusoid. PloS one 9 (11), e111864-e111864.

47. Jeong, G.S. et al. (2016) Viscoelastic lithography for fabricating self-organizing soft microhoneycomb structures with ultra-high aspect ratios. Nature Communications 7, 11269.

48. Stucki, J.D. et al. (2018) Medium throughput breathing human primary cell alveolus-on-chip model. Scientific reports 8 (1), 14359-14359.

49. Sundarakrishnan, A. et al. (2018) Engineered cell and tissue models of pulmonary fibrosis. Advanced Drug Delivery Reviews 129, 78-94.

50. Sundarakrishnan, A. et al. (2019) Bioengineered in Vitro Tissue Model of Fibroblast Activation for Modeling Pulmonary Fibrosis. ACS Biomaterials Science \& Engineering 5 (5), 2417-2429.

51. Zhou, M. et al. (2014) Induction of epithelial-to-mesenchymal transition in proximal tubular epithelial cells on microfluidic devices. Biomaterials 35 (5), 1390-1401.

52. Kim, S. et al. (2016) Pharmacokinetic profile that reduces nephrotoxicity of gentamicin in a perfused kidney-on-a-chip. Biofabrication 8 (1), 015021.

53. Rouwkema, J. and Khademhosseini, A. (2016) Vascularization and Angiogenesis in Tissue Engineering: Beyond Creating Static Networks. Trends in Biotechnology 34 (9), 733-745.

54. Zhu, W. et al. (2017) Direct 3D bioprinting of prevascularized tissue constructs with complex microarchitecture. Biomaterials 124, 106-115.

55. Kolesky, D.B. et al. (2016) Three-dimensional bioprinting of thick vascularized tissues. Proceedings of the National Academy of Sciences 113 (12), 3179.

56. Homan, K.A. et al. (2019) Flow-enhanced vascularization and maturation of kidney organoids in vitro. Nature Methods 16 (3), 255-262.

57. Lin, N.Y.C. et al. (2019) Renal reabsorption in 3D vascularized proximal tubule models. Proceedings of the National Academy of Sciences 116 (12), 5399.

58. Jin, Y. et al. (2018) Vascularized Liver Organoids Generated Using Induced Hepatic Tissue and Dynamic Liver-Specific Microenvironment as a Drug Testing Platform. Advanced Functional Materials 28 (37), 1801954.

59. Kurokawa, Y.K. et al. (2017) Human Induced Pluripotent Stem Cell-Derived Endothelial Cells for Three-Dimensional Microphysiological Systems. Tissue engineering. Part C, Methods 23 (8), 474484.

60. van der Meer, A.D. et al. (2013) Three-dimensional co-cultures of human endothelial cells and embryonic stem cell-derived pericytes inside a microfluidic device. Lab on a Chip 13 (18), 3562-3568.

61. Veerman, C.C. et al. (2015) Immaturity of Human Stem-Cell-Derived Cardiomyocytes in Culture: Fatal Flaw or Soluble Problem? Stem Cells and Development 24 (9), 1035-1052. 
62. Krumdieck, C.L. et al. (1980) A new instrument for the rapid preparation of tissue slices. Analytical Biochemistry 104 (1), 118-123.

63. Vickers, A.E.M. et al. (2004) Organ Slice Viability Extended for Pathway Characterization: An in Vitro Model to Investigate Fibrosis. Toxicological Sciences 82 (2), 534-544.

64. Alsafadi, H.N. et al. (2017) An ex vivo model to induce early fibrosis-like changes in human precision-cut lung slices. American Journal of Physiology-Lung Cellular and Molecular Physiology 312 (6), L896-L902.

65. Stribos, E.G.D. et al. (2016) Precision-cut human kidney slices as a model to elucidate the process of renal fibrosis. Translational Research 170, 8-16.e1.

66. Paish, H.L. et al. (2019) A Bioreactor Technology for Modeling Fibrosis in Human and Rodent Precision-Cut Liver Slices. Hepatology 70 (4), 1377-1391.

67. Sun, H. et al. (2016) Netrin-1 Regulates Fibrocyte Accumulation in the Decellularized Fibrotic Sclerodermatous Lung Microenvironment and in Bleomycin-Induced Pulmonary Fibrosis. Arthritis \& Rheumatology 68 (5), 1251-1261.

68. Booth, A.J. et al. (2012) Acellular normal and fibrotic human lung matrices as a culture system for in vitro investigation. American journal of respiratory and critical care medicine 186 (9), 866876.

69. van Wenum, M. et al. (2014) Bioartificial livers in vitro and in vivo: tailoring biocomponents to the expanding variety of applications. Expert Opinion on Biological Therapy 14 (12), 1745-1760.

70. Hinz, B. et al. (2012) Recent developments in myofibroblast biology: paradigms for connective tissue remodeling. The American journal of pathology 180 (4), 1340-1355.

71. Yazdani, S. et al. (2017) Drug targeting to myofibroblasts: Implications for fibrosis and cancer. Advanced Drug Delivery Reviews 121, 101-116.

72. Hu, B. and Phan, S.H. (2013) Myofibroblasts. Current opinion in rheumatology 25 (1), 71-77.

73. Iwaisako, K. et al. (2014) Origin of myofibroblasts in the fibrotic liver in mice. Proceedings of the National Academy of Sciences of the United States of America 111 (32), E3297-E3305.

74. Lepreux, S. and Desmoulière, A. (2015) Human liver myofibroblasts during development and diseases with a focus on portal (myo)fibroblasts. Frontiers in physiology 6, 173-173.

75. Wynn, T.A. (2011) Integrating mechanisms of pulmonary fibrosis. The Journal of experimental medicine 208 (7), 1339-1350.

76. Kim, M.Y. et al. (2010) Hemodynamic alterations in cirrhosis and portal hypertension. The Korean journal of hepatology 16 (4), 347-352.

77. Kis, K. et al. (2011) Myofibroblast differentiation and survival in fibrotic disease. Expert reviews in molecular medicine 13, e27-e27.

78. Wells, R.G. (2013) Tissue mechanics and fibrosis. Biochimica et biophysica acta 1832 (7), 884890.

79. Sandbo, N. et al. (2016) Control of myofibroblast differentiation and function by cytoskeletal signaling. Biochemistry (Moscow) 81 (13), 1698-1708.

80. Tschumperlin, D.J. et al. (2018) Mechanosensing and fibrosis. J Clin Invest 128 (1), 74-84. 
